\title{
Mining Lake Time Series using Symbolic Representation
}

\author{
Guangchen Ruan ${ }^{\mathrm{a}, *}$, Paul C. Hanson ${ }^{\mathrm{b}}$, Hilary A. Dugan ${ }^{\mathrm{b}}$, Beth Plale ${ }^{\mathrm{a}}$ \\ ${ }^{a}$ School of Informatics and Computing, Indiana University, 919 E. 10th Street, \\ Bloomington, IN 47408, USA \\ ${ }^{b}$ Center for Limnology, University of Wisconsin-Madison, 680 North Park Street, Madison, \\ WI 53706, USA
}

\begin{abstract}
Sensor networks deployed in lakes and reservoirs, when combined with simulation models and expert knowledge from the global community, are creating deeper understanding of the ecological dynamics of lakes. However, the amount of data and the complex patterns in the data demand substantial compute resources and efficient data mining algorithms, both of which are beyond the realm of traditional limnological research. This paper uniquely adapts methods from computer science for application to data intensive ecological questions, in order to provide ecologists with approachable methodology to facilitate knowledge discovery in lake ecology. We apply a state-of-the-art time series mining technique based on symbolic representation (SAX) to high-frequency time series of phycocyanin (PHYCO) and chlorophyll (CHLORO) fluorescence, both of which are indicators of algal biomass in lakes, as well as model predictions of algal biomass (MODEL). We use data mining techniques to demonstrate that MODEL predicts PHYCO better than it predicts CHLORO. All time series have high redundancy, resulting in a relatively small subset of unique patterns. However, MODEL is much less complex than either PHYCO or CHLORO and fails to reproduce high biomass periods indicative of algal blooms. We develop a set of tools in $\mathrm{R}$ to enable motif discovery and anomaly detection within a
\end{abstract}

\footnotetext{
* Corresponding author

Email addresses: gruan@indiana.edu (Guangchen Ruan), pchanson@wisc.edu (Paul C.

Hanson), hdugan@wisc.edu (Hilary A. Dugan), plale@indiana.edu (Beth Plale)
}

Preprint submitted to Ecological Informatics

March 6, 2017

(C) 2016. This manuscript version is made available under the Elsevier user license http://www.elsevier.com/open-access/userlicense/1.0/ 
single lake time series, and relationship study amongst multiple lake time series through distance metrics, clustering and classification. Furthermore, to improve computation times, we provision web services to launch $\mathrm{R}$ tools remotely on high performance computing (HPC) resources. Comprehensive experimental results on observational and simulated lake data demonstrate the effectiveness of our approach.

Keywords: lake time series, symbolic representation, mining

\section{Introduction}

Ecology has entered an era of "big data" in which the necessities for managing and analyzing data are inspiring scientists to develop novel approaches to use those data for answering contemporary ecological questions [1]. Data from

5 aquatic sensor networks are a good example of how observations for limnological variables, such as water temperature, dissolved oxygen, and phytoplankton biomass, have grown in volume. A few decades ago, one year of these data may have included hundreds of manually collected measurements; whereas, today, those same variables are sampled by autonomous sensors at frequencies up to one hertz, resulting in $0.5 \mathrm{M}$ measurements per year per variable [2, 3]. When data from multiple depths, multiple years and multiple observatories are combined, the number of recorded measurements can reach into the billions. Data of these volumes stress the computational capacity of most desktop computers, and data analysis software commonly used by ecologists slows considerably when handling extremely large data sets. Even common data manipulations, such as searches, sorts, and sub-setting, become unacceptably slow.

Representation of time series data in ways that reduces the volume of data while retaining pattern relevant to ecological questions, would help ecologists overcome data volume as an obstacle to data analysis. In the field of computer science, symbolic representation of time series data was developed to help solve this problem. Specifically, Symbolic Aggregate approXimation, or SAX [4], was developed to transform time series data into symbols, as has been used 
in a variety of domains, such as acoustics, medicine, and image analysis [5, 6]. Symbols not only require less space to store, but also retain essential statistical characteristics of the original time series and are amenable to machine learning algorithms designed for natural language processing [4]. SAX has proven to perform as well as, or even better than, original representation of data for use in common data mining techniques, such as clustering, anomaly detection, classification, and motif discover 7]. Despite these advantages, we are unaware of its implementation in ecology.

Here, we introduce SAX as an approach to analyzing chlorophyll and phycocyanin (both light harvesting pigments) data from high frequency lake sensors and lake algal biomass predicted from a simulation model. We demonstrate how the SAX transformation greatly reduces data volume. And we adapt pattern 35 detection and classification algorithms from computer science as first steps in addressing the following questions: What patterns exist in lake chlorophyll and phycocyanin data? Are the diversity and frequency of occurrence of patterns in fluorescence data matched by the patterns in predictions from lake simulations? What do the differences between observed and predicted variables tell us about lakes and the models we use to predict their time dynamics?

With SAX, we developed a set of $\mathrm{R}$ tools to conduct motif discovery [8] and anomaly detection [9, 10] within a single lake time series, and relationship study amongst multiple lake time series through distance metrics, clustering and classification. We conduct comprehensive experiments on observational and simulated data to demonstrate the effectiveness of our tools.

The remainder of this paper is organized as follows: Section 2 briefly describes the methodology of representing time series as symbolic characters, which serves as the foundation of mining algorithms to be developed. In Section 3. we present overall architecture and detail mining algorithms. Section 4 presents comprehensive experiments on observational and simulated lake time series data to validate the effectiveness of our approach. Related work is given in Section 5 Finally, we conclude the paper in Section 6 . 


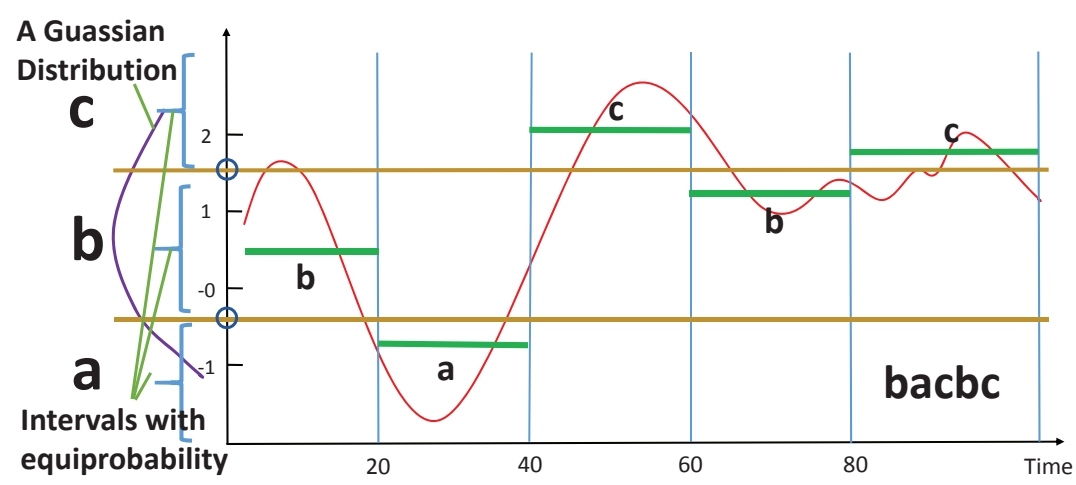

Figure 1: Representing time series with Symbolic Aggregate approXimation (SAX). In this example, the $\mathrm{x}$-axis of the time series (red line) is divided into five frames. The mean value of the data in each frame is represented by a symbol on the y-axis. The resulting letters form a word, in this case 'bacbc'.

\section{Symbolic Aggregation Approximation}

The challenges outlined in Section 1 all require a robust and efficient time series representation for which a rigorous distance metric can be defined. While there are many useful representations of time series data 11, 12, 13, 14, 15, 16], Symbolic Aggregate approXimation or SAX [4], a symbolic representation of time series, solves many algorithmic problems through dimensionality and volume reduction, quantification of similarity between time series, and discovery and representation of otherwise difficult to handle data features, such as highly skewed probability densities. Essentially, SAX converts data to words and uses natural language processing algorithms to discover and classify patterns and collections of patterns in a manner akin to developing a dictionary (i.e., the possible words) and a vocabulary (i.e., the dictionary subset used in a time series). For example, a rare word in sensor data that might represent a surprising algal bloom can be catalogued and easily searched within the collection of words created by the SAX representation of the simulated data.

We use Figure 1 to briefly illustrate how SAX works. The first phase of SAX is to apply Piecewise Aggregate Approximation (PAA) dimensionality reduction [14] where the original time series $C$ of length $n$ is reduced into a $w$ - 
dimensional space as a vector $\bar{C}=\bar{c}_{1}, \ldots, \bar{c}_{w}$. The $i^{\text {th }}$ element of $C$ is calculated by Eq. 1]

$$
\bar{c}_{i}=\frac{w}{n} \sum_{j=\frac{n}{w}(i-1)+1}^{\frac{n}{w} i} c_{j}
$$

Simply stated, to reduce the time series from $n$ dimensions to $w$ dimensions, the data is divided into $w$ equal sized "frames" (a frame shown as a segment within two consecutive blue vertical lines as in Figure 1). The mean value of the data falling within a frame is calculated (i.e. the PAA segments shown as horizontal green bars as in Figure 1) and a vector of these values becomes the data-reduced representation.

In the second phase, SAX applies a further transformation which produces symbols/characters with equiprobability to obtain a discrete representation. This can be achieved since normalized time series have a Gaussian distribution and we can simply determine the "breakpoints" that will produce a equalsized areas under Gaussian curve by looking up a statistical table [17]. We in turn map each interval to a symbol ('a', 'b', and 'c' bottom up as in Figure 1) 85 and accordingly a PAA segment is represented by the symbol of the interval it falls into. At this stage, we finish transforming a time series into a SAX word ('bacbc' in this case). The number of symbols in a word is called word size and the number of possible symbols (i.e., the number of equiprobable intervals) is called alphabet size. With these two concepts, we can determine the number of possible words, i.e., "vocabulary size" to be $a^{w}$, where a is alphabet size and w is word size. One potential concern of SAX is that each serial transformation step might be introducing some bias, making the knowledge (e.g., discovered patterns) interpretation harder. However, we believe that the benefits introduced by the two processes, i.e., dimensionality reduction, lower bounding and volume reduction outweigh the disadvantages incurred by bias, as demonstrated in [4] that SAX yields better performance than the original data for data mining tasks such as classification, clustering and etc. 


$$
\operatorname{MINDIST}(\hat{Q}, \hat{C}) \equiv \sqrt{\frac{n}{w}} \sqrt{\sum_{i=1}^{w}\left(\operatorname{dist}\left(\hat{q}_{i}, \hat{c}_{i}\right)\right)^{2}}
$$

Apart from dimensionality and volume reduction, another promising feature of SAX is that a distance measure can be defined on a SAX word. The dis-

100 tance measure on a SAX word, gives a lower bound of corresponding distance measure (e.g. Euclidean distance) defined on the original series. Eq. 2 defines the MINDIST function that returns the minimum distance between the two transformed SAX words $\hat{Q}$ and $\hat{C}$, where $\hat{q}_{i}$ and $\hat{c}_{i}$ are the transformed symbols (e.g., 'a' and 'c'). The coefficient $\sqrt{\frac{n}{w}}$ in Eq. 2 can be viewed as the compression ratio. The value of the distance function $\operatorname{dist}\left(\hat{q}_{i}, \hat{c}_{i}\right)$ can be obtained by looking up the statistical table [17].

Limitation.. We consider following limitations when applying SAX-based transformation: (1) Decrease in volume means potentially throwing out useful patterns at shorter time scales. To address this issue, an variant of SAX called iSAX [18] was developed for multi-scale transformation. (2) Because SAX deals with normalized data, it may lose the ability to calculate fluxes or compare mass balances, both of which require data in their original units. This also means analyses tend to be correlative in nature rather than process-oriented. (3) SAX is more designed for univariate time series. predicting one SAX time series from a suite of possible predictor SAX time series (e.g., multiple time series that showing covariances and process dependencies) has gone unexplored in this manuscript.

\section{Lake Time Series Mining System}

\subsection{Overall Architecture}

The overall design philosophy of our lake time series mining system is to interface users (i.e., ecologists) with simple and convenient analytical environment, e.g., composing scripts with provisioned library functions, and in the 
meantime hiding scalable computing details with the implementation of the tools. To this end the architecture of our lake time series mining system is influenced by several factors. First, the limnology community has comfort with R, making that tool a good choice. Second, High Performance Computing (HPC) resources are not places to carry out development because the model of use is batch oriented. Finally, we make the presumption that the ability to analyze large $(>100 \mathrm{MB})$ time series datasets using text and data mining techniques is useful new functionality for the limnology community. The architecture, shown as a cartoon diagram in Figure 2, is implemented as a set of $\mathrm{R}$ libraries 1 . The $\mathrm{R}$ libraries carry out a set of classic time series mining tasks, including quantified distance measure, motif discovery, anomaly detection, clustering, and classification, each of which will be further described in detail in Section 3.2. The model of use is for lake ecologists to start by debugging their analysis process on a small dataset, then running on the HPC resources (i.e., IU BigRed II [19]). The debugging is done from within their favourite IDE (e.g., RStudio) on their desktop. Once ecologists verify the analytical results locally, they are ready for large-scale remote simulation. To satisfy this need, we develop web services (in particular RESTful services) to support simple job submission on HPC or cloud resources with much more powerful computing capability than a single machine. The web service calls are designed to act in a manner asynchronous to the user's desktop so that users are not blocked by the invocation.

\subsection{Mining Algorithms}

145

We describe the suite of mining algorithms in this section before moving to their evaluation in Section 4 .

\subsubsection{Time Series Alignment}

It is common for time series from in situ sensors to have different sampling rates and clocks that are out of synchronization (i.e., a time point may not be

\footnotetext{
${ }^{1}$ Our libraries are publicly accessible at https://github.com/Data-to-Insight-Center/laketimeseries-mining
} 


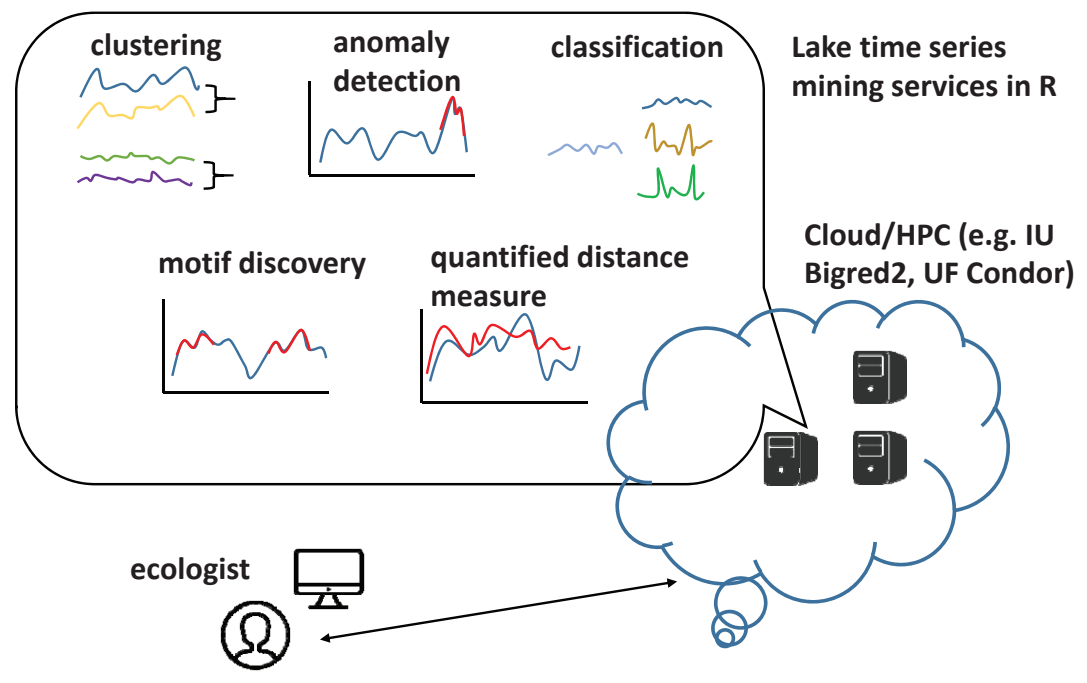

Figure 2: Architecture sketch of lake time series mining system

150

recorded as the same timestamp on different sensors). Hence, preprocessing is necessary before any comparison can be made. Our tool suite includes time series alignment.

For the case that sensors have different sampling rates (i.e. time resolution) but with synchronized clocks, we align time series by interpolation, which can either be linear or non-linear (e.g., spline interpolation [20] where the interpolant is a special type of piecewise polynomial called a spline).

For the more complicated case where both sampling rate and internal time are different, we employ a two-phase approach where the first step is to use aforementioned interpolation to make a matching time resolution, followed by a time alignment with Dynamic Time Warping (DTW, as shown in Figure 3(b)] 21], which allows a non-linear (elastic) alignment and produces better similarity score than linear time alignment (Figure 3(a)p. We further extend DTW to application on transformed SAX words rather than raw time series, and simply use the distance function $\operatorname{dist}()$ in Eq. 2 as the pairwise distance measure of SAX symbols, with the restriction that two SAX words to be aligned should be of the same length. 


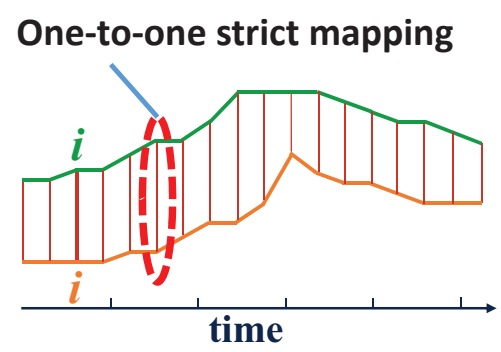

(a) Linear time alignment.

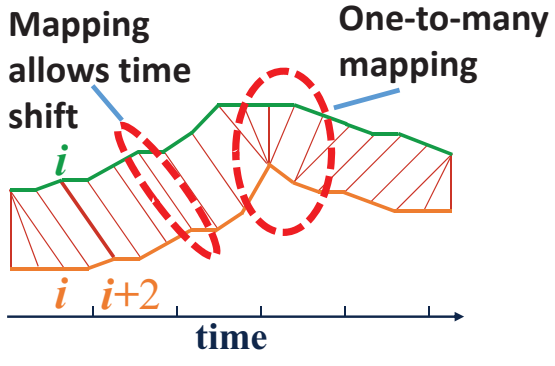

(b) Non-linear time alignment.

Figure 3: Linear versus non-linear time alignment (i.e., dynamic time warping) of time series.

\subsubsection{Time Series Slicing}

After alignment of raw time series, each time series must be normalized to have a mean of zero and a standard deviation of one before converting it to compare time series with different offsets and amplitudes [22].

As suggested in [4], oftentimes it is meaningless to directly transform a long raw time series into a single SAX word. Instead, the time series is sliced into multiple subsequences each of which is in turn symbolized as a SAX word. 


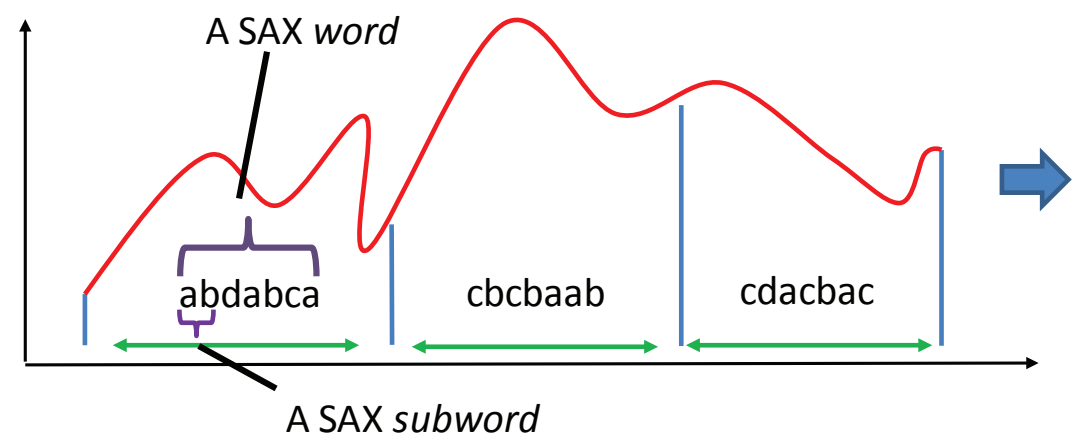

(a)

\begin{tabular}{|l|l|l|l|}
\hline aa & ab & ba & bb \\
\hline ac & ad & bc & bd \\
\hline ca & cb & da & db \\
\hline cc & cd & dc & dd \\
\hline
\end{tabular}

(b)

\begin{tabular}{|l|l|l|l|}
\hline 1 & 3 & 2 & 0 \\
\hline 3 & 0 & 2 & 1 \\
\hline 1 & 2 & 1 & 1 \\
\hline 0 & 2 & 0 & 0 \\
\hline
\end{tabular}

(c)

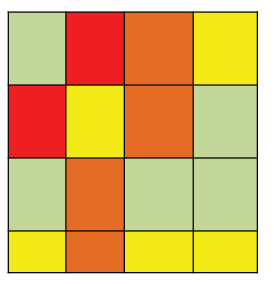

(d)

Figure 4: Transformation of a time series into a Time Series Bitmap (TSB). a) Each SAX word is further divided into subwords using a sliding window of two letters. b) A matrix is constructed showing all possible subwords. c) A numerical matrix is constructed representing the frequency of each subword in the time series. d) The numerical matrix is visualized as a colored bitmap. 
Figure 5. two consecutive TSBs are compared to detect whether an anomaly has occurred, as to be discussed shortly in Section 3.2.5

We use Figure 4 to illustrate the principle. As shown in Figure 4(a), we still chunk the raw time series into subsequences each of which is transformed into a SAX word. In this case, we get SAX words "abdabca", "cbcbaab", "cdacbac" and etc with a non-overlapping sliding window. For each SAX word, we in turn use an overlapping sliding window to generate SAX subwords. For instance, with subword size of two, SAX word "abdabca" is sliced into subwords " $a b "$, " $b d "$, " $d a "$, " $a b "$, " $b c$ " and "ca". Based on this, we further use a matrix to represent the frequency of each possible subword, as shown in Figures 4(b) and 4(c). Since the time series in a data collection may be of various lengths, we normalize the frequencies by dividing by the largest value. Hence the value of each cell in the matrix is from 0 to 1 . After normalization, we can easily visualize the numerical matrix as a bitmap, as shown in Figures 4(d), providing a intuitive means to examine the similarity of time series. In summary, TSB is a global summary of local shapes.

We remove the restriction in the original approach in [10] which requires the matrix to be square and even number of rows/columns, by allowing the matrix to be any shape. To be general, we treat the matrix as a one dimensional array with $v^{s}$ cells/entries, where $v$ is the aforementioned alphabet size and $s$ is the subword size. For visualization, we just reshape the 1-D array to a matrix whose shape is closest to a square. The criterion we used can be formally expressed as $\arg \min a b s \mid$ nrows - ncols $\mid$.

\subsubsection{Quantified Distance Measure}

To systematically measure the similarity or difference of time series, we need quantified distance metrics. SAX has its own built-in metric, as shown in Eq. 2 for SAX words or subsequences. Since TSB in essence is a 1-D numerical array, a plethora of well defined distance metrics are available off the shelf, e.g., "manhattan", "euclidean", "maximum", etc. Here we note that unlike distance metrics on numerical arrays, SAX's built-in distance is only a pairwise distance 
metric where operations like "addition", "substraction", "multiplication" and "division" are missing. This can put restrictions on algorithms that can be used for clustering and classification, as we will discuss subsequently in Section 3.2.7

$$
C D M(x, y)=\frac{C(x y)}{C(x)+C(y)}
$$

In this section we focus on discussing another very useful distance measure called Compression-Based Dissimilarity Measure or CDM [9], which is based on the concept of Kolmogorov complexity [23]. Kolmogorov complexity is a measure of randomness of strings (in our case symbolized SAX words) based on their information content. The Kolmogorov complexity $K(x)$ of a string $x$ is defined as the length of the shortest program capable of producing $x$ on a universal computer - such as a Turing machine. Inspired by its idea, we define $C(x)$ as the length of the resulting compressed string with the best combination of compression tool and compression parameters for string $x$. We note that the optimal compression tool and compression parameters should be used to get the best approximation of the Kolmogorov complexity. In practice, we simply try various compression algorithms (e.g., "gzip", "bzip2", and "xz") and parameters and use the one producing the best compression ratio.

The formal CDM distance measure is given by Eq. 3, it is close to 1 when $x$ and $y$ are not related, and smaller than one if $x$ and $y$ are related. The smaller the $C D M(x, y)$, the more closely related $x$ and $y$ are. Note that $C D M(x, x)$ is not zero.

\subsubsection{Anomaly Detection}

To perform anomaly detection, there are two approaches, one based on CDM [9] and the other based on TSB [10]. For CDM-based approach, we use a divide-and-conquer strategy. The idea is as follows: both the left and right halves of the entire sequence being examined are compared to the entire sequence using the CDM dissimilarity measure. The intuition is that the side containing the most unusual section will be less similar to the global se- 
quence than the other half. Having identified the most interesting side, we can recursively repeat the above process, repeatedly dividing the most interesting section until we can no longer divide the sequence. Algorithm 1 summarizes this procedure.

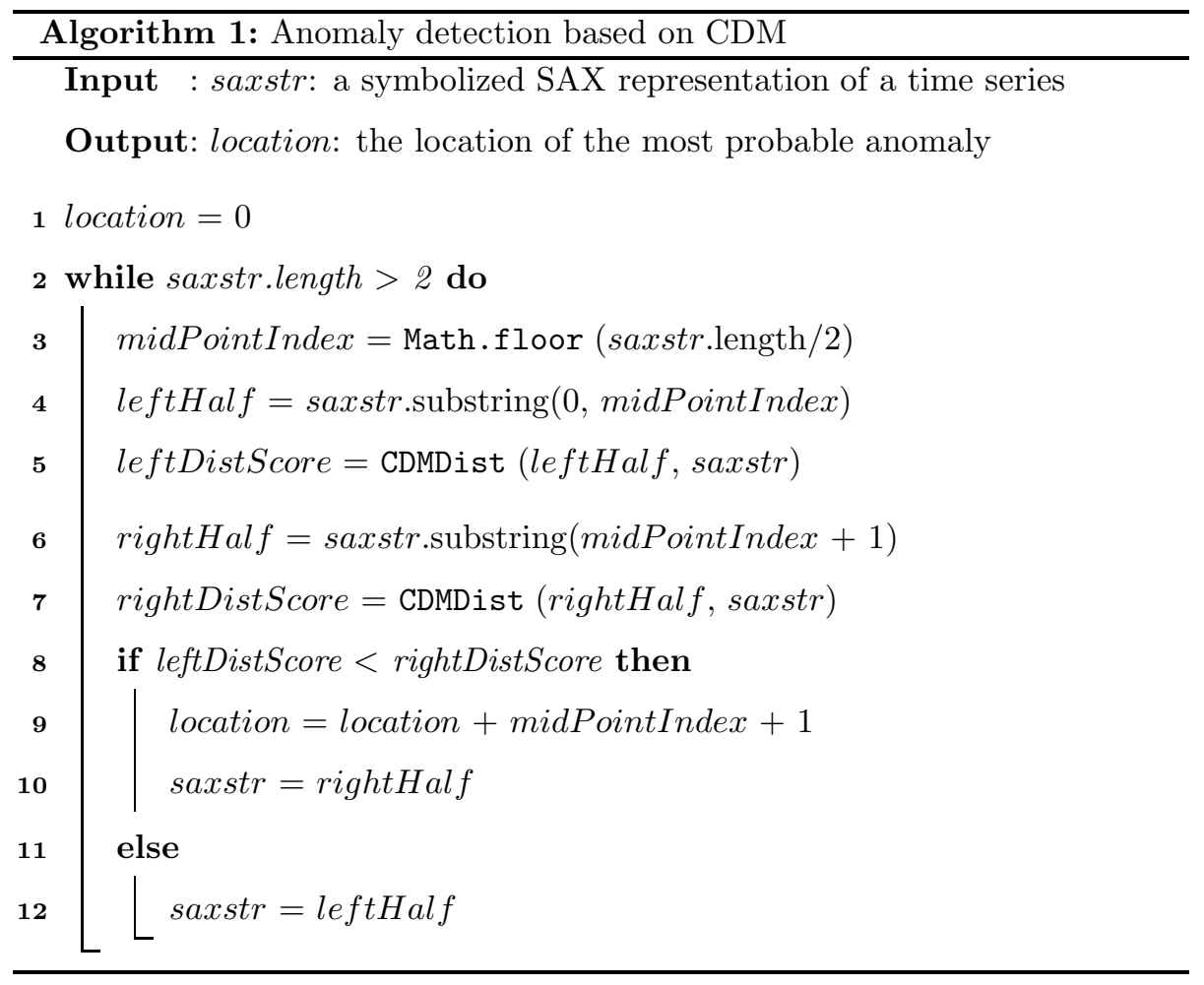

This approach, however, has two limitations. First, it assumes a single anomaly in the SAX string. Second, in the first few iterations, the measure needs to note the difference a small anomaly makes, even when masked by a large amount of surrounding normal data. To overcome these limitations, we can divide the input sequence into $W$ contiguous sections/windows, and assign the anomaly value of the $i t h$ window as $C D M\left(W_{i}\right.$, saxstr $)$. In other words, we simply measure how well a small local section can match the global sequence.

255 TSB based approach is able to detect multiple anomalies with following idea: we create two consecutive windows, and slide them together across the 


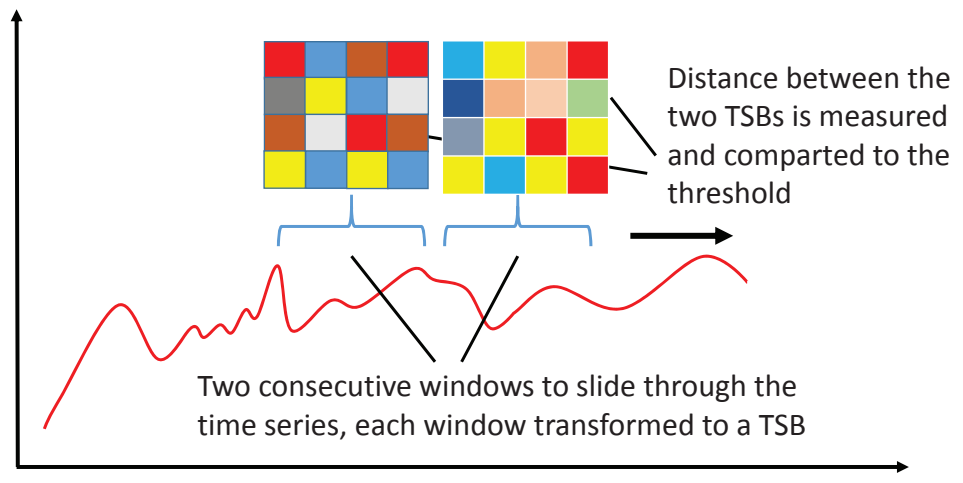

Figure 5: Anomaly detection with time series bitmap.

sequence. At each time instance we build a time series bitmap each of the two windows, and measure the distance between them. This distance we report as an anomaly score. When this score is greater than the pre-specified threshold, we think there is an anomaly. This process is illustrated by Figure 5

\subsubsection{Motif Discovery}

To perform motif finding, we can adopt the approach in [8]. The goal is find the most frequent subsequences from within a time series. When the subsequences are sliced through an overlapping window, we discard trivial matches which are defined as matches immediately before and after the subsequence in question, as shown in Figure6. This process can be significantly inefficient since the time complexity of excluding trivial matches is quadratic. However, this is not a problem in the domain of lake time series since the subsequences we examine are extracted by a non-overlapping sliding window. Moreover, we note that subsequences are transformed into SAX words based on which frequency is calculated. As shown in Figure 1. PAA segments falling into the same interval are represented by the same symbol, hence a SAX word is actually a signature representing a group of similar subsequences being symbolized. Therefore, no extra effort is needed to consider approximates of a given subsequence when it is in a numerical form. 


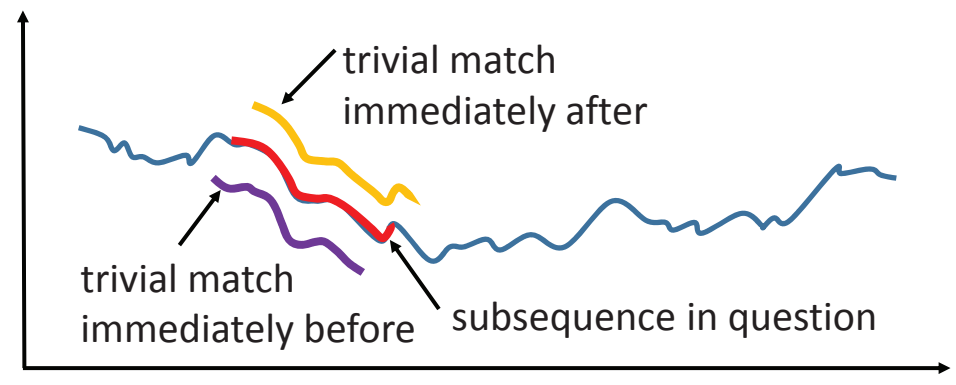

Figure 6: Trivial match in motif finding.

\subsubsection{Clustering and Classification}

Depending on the distance metric defined on the representation of the time series, different clustering algorithms can be used. For clustering algorithms that only require pairwise distance (e.g., hierarchical clustering), we can use either SAX or TSB representation. For clustering algorithms that require a complete distance metric (e.g., KMeans clustering), only TSB can be used since the "addition" and "division" operations that are used to average the points belonging to the same group membership in each iteration to derive the new centroid are not defined on SAX words.

The above statement also holds for classification algorithms. In lake time series study, we heavily use $k$ nearest neighbor which is a simple yet effective unsupervised algorithm. Since it only requires pairwise distance, both SAX and TSB are applicable. We will see more examples of clustering and classification in Section 4

\section{Experiments}

High algal biomass in lakes can lead to a serious deterioration in water quality, and therefore understanding and predicting algal biomass patterns are fundamentally important to lake management. While mean phytoplankton concentrations are predicted well by lake phosphorous concentration [24], and sea- 

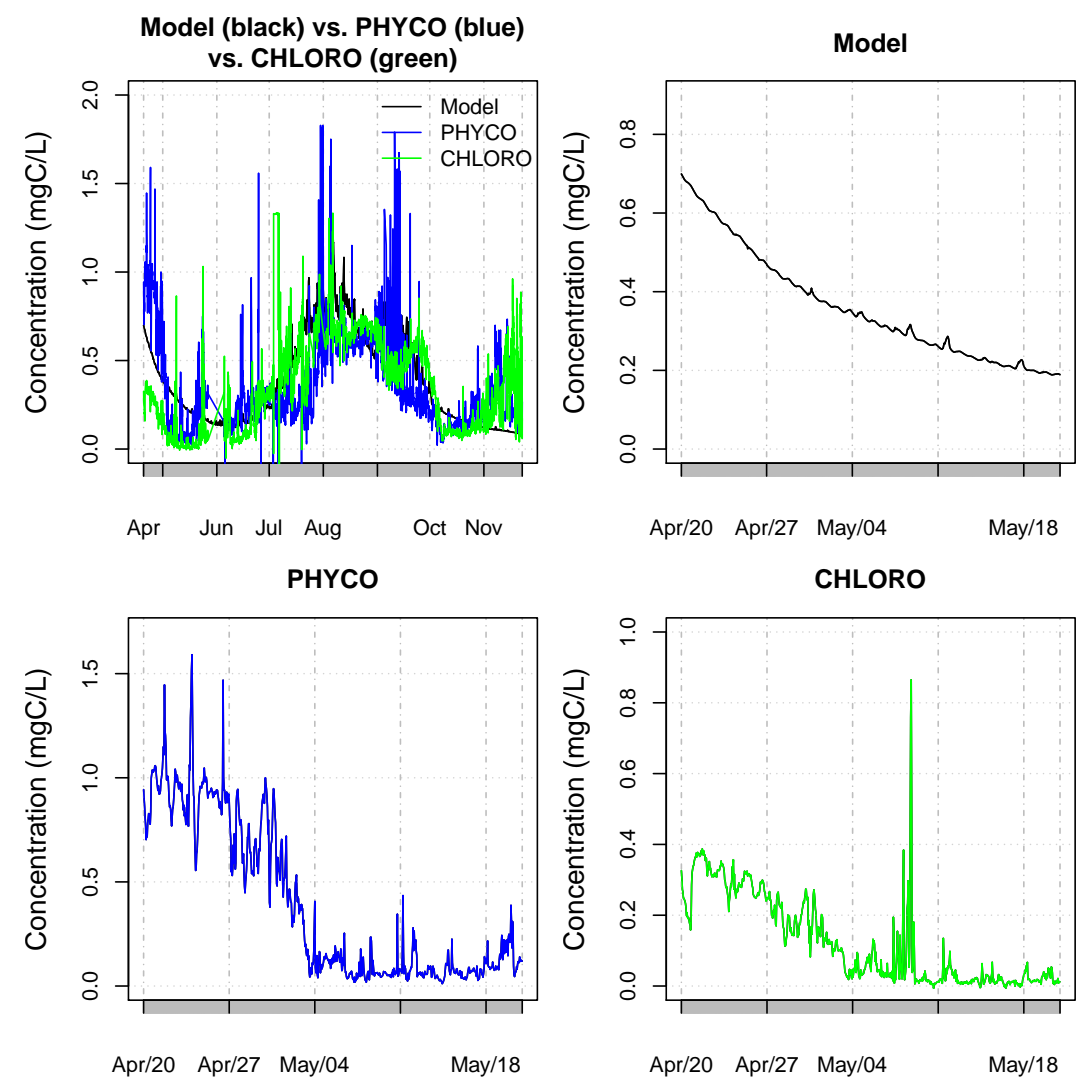

Figure 7: Lake time series: Model, PHYCO and CHLORO. a) Time series of phycocyanin (blue), chlorophyll (green), and modeled phytoplankton concentration (black) in Lake Mendota for 2009. b-d) The x-axis has been shorted to five weeks to daily variability between the three time series.

high uncertainty remains over shorter time scales of hours to days. New data from high frequency sensor networks and predictions from new numerical simulations [26] provide an opportunity to discover higher frequency patterns in lake phytoplankton. However, ecologists lack quantitative methods to investigate the efficacy of models in recreating high frequency patterns. The efficacies a model may depend on the dominant phytoplankton group in the lake, how well the model represents ecosystem processes controlling phytoplankton growth and death, and perhaps most importantly, the time scale of the model [27]. 
For our experiment using SAX, we chose three time series that represent for quantitative comparison of patterns between our time series. Results show the occurrence of prevalent word clusters (Fig. 9), and that both PHYCO and CHLORO have a wider distribution of vocabulary. Importantly, the motifs that 


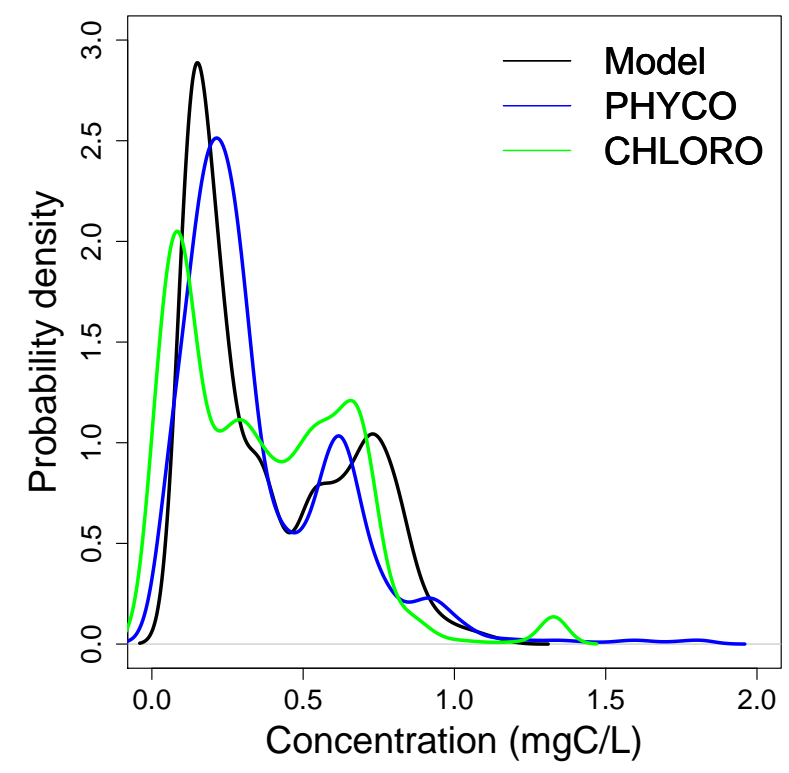

Figure 8: Probability density of Model, PHYCO and CHLORO

335 cases, the dominant motif is a pattern that begins with low algal biomass and rises through to a maximum in the evening. The second most frequent motif is a pattern that begins at moderate biomass concentrations, drops throughout the morning, and rises in the afternoon or evening. a single SAX string because we are interested in identifying the prevalence of patterns that occur at a daily time scale. To further compare time series in its entirety, we transform a time series into a Time Series Bitmap (TSB) which can be regarded as a compact summarization of the entire time series. We can treat a probability density as a time series and apply TSB as well. Figures 11(a) to 11(c) show the TSB of "CHLORO and PHYCO", "CHLORO and MODEL" and "PHYCO and MODEL", respectively. In each figure, the top panel shows the TSB of raw time series, the bottom panel shows the TSB for its probability density. The subword size is set to 2 . For probability density, we increase the 

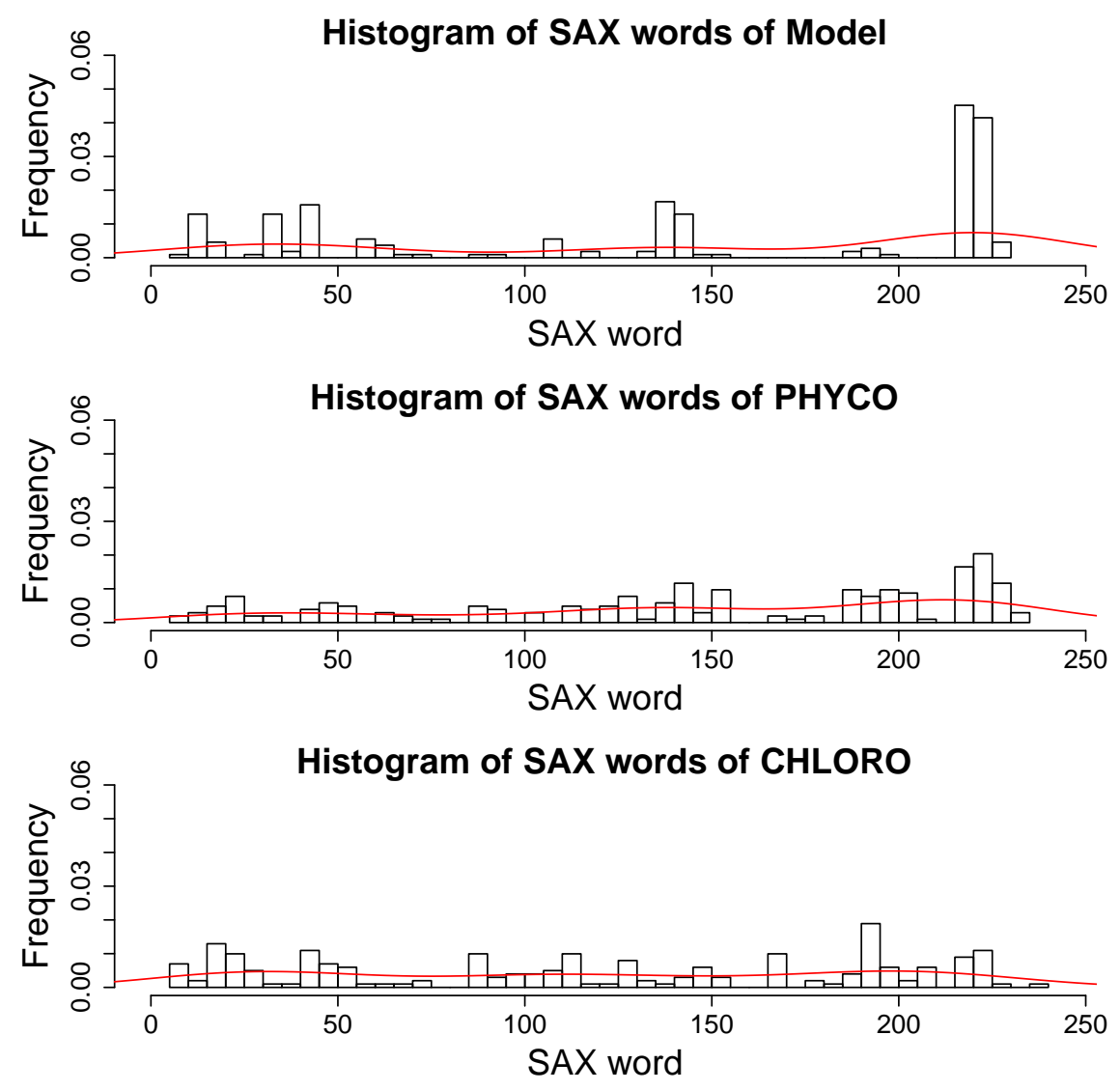

Figure 9: Histogram of SAX words of Model, PHYCO and CHLORO. Red line shows kernel density estimates. 


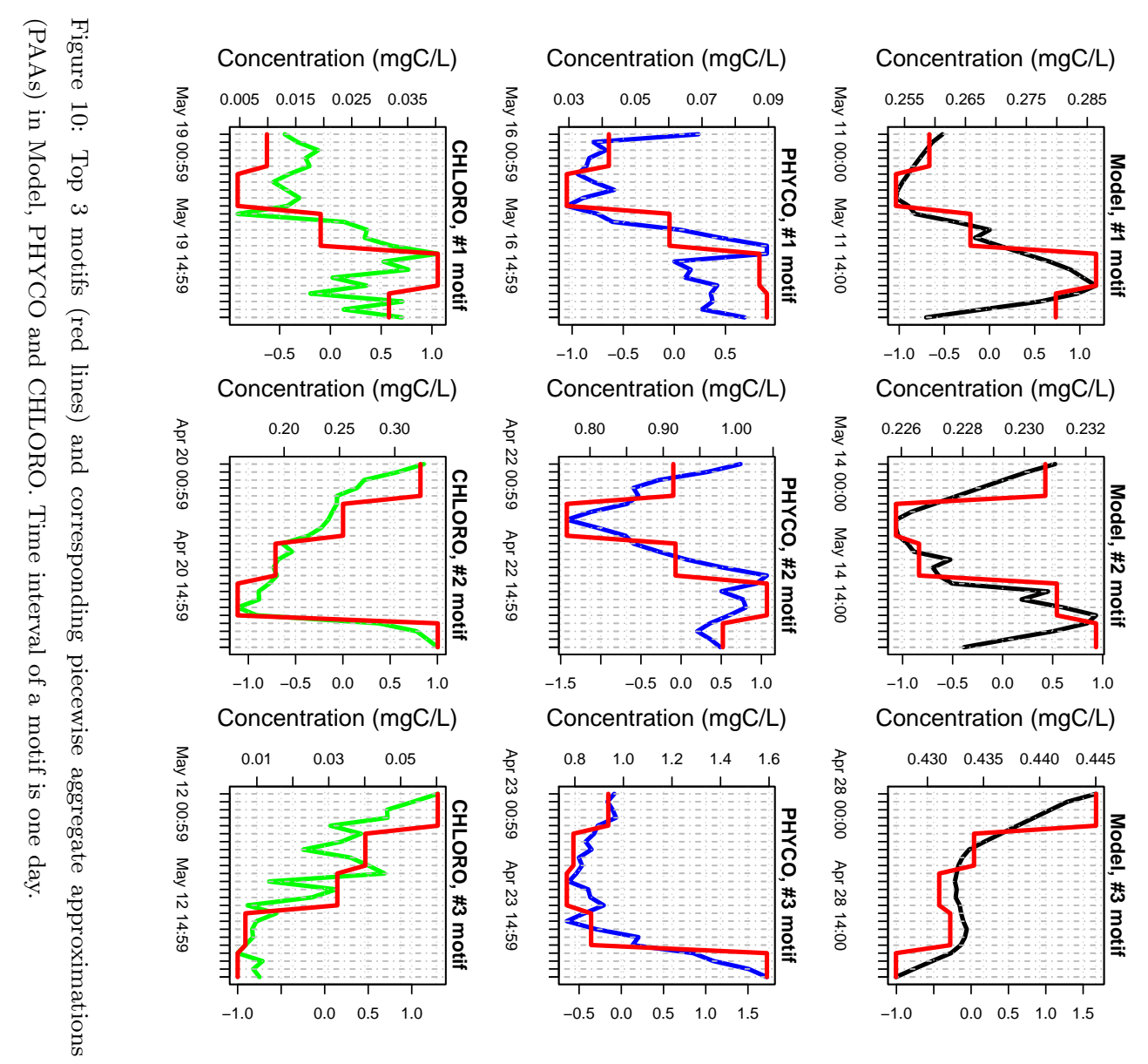


Table 1: Distance matrix of Model, PHYCO, CHLORO and Random, measured by TSB.

\begin{tabular}{|c|c|c|c|}
\hline & Model & PHYCO & CHLORO \\
\hline PHYCO & 0.258 & & \\
\hline CHLORO & 0.294 & 0.339 & \\
\hline Random & 1.591 & 1.610 & 1.479 \\
\hline
\end{tabular}
sequence also has fewer unique words that CHLORO and PHYCO.

Which time series are most similar? We found it encouraging that MODEL and PHYCO were most similar, followed closely by MODEL and CHLORO 


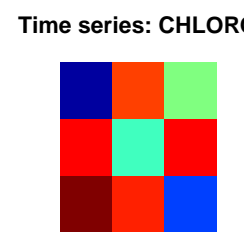

Probability density of time series: CHLORO
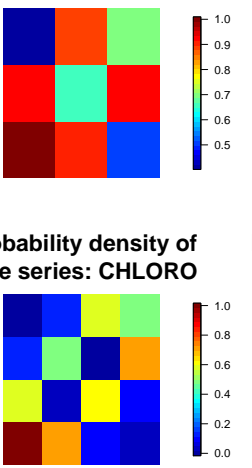

Time series: PHYCO

Probability density of time series: PHYCO

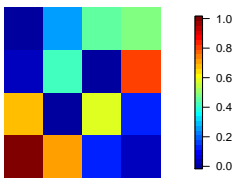

(a) CHLORO vs. PHYCO

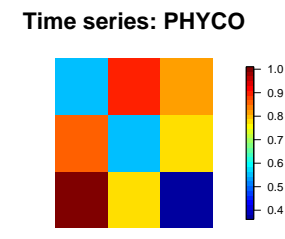

Probability density of time series: PHYCO

Probability density of
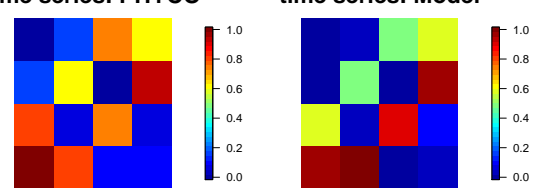

(c) PHYCO vs. Model.

Figure 11: Time series bitmap (TSB) comparison. Top panel is TSB for raw time series and bottom panel is TSB for corresponding probability distribution.

Table 2: Distance matrix of probability density of Model, PHYCO, CHLORO and Random, measured by TSB

\begin{tabular}{|c|c|c|c|}
\hline & Model & PHYCO & CHLORO \\
\hline PHYCO & 0.481 & & \\
\hline CHLORO & 0.441 & 0.257 & \\
\hline Random & 0.540 & 0.469 & 0.486 \\
\hline
\end{tabular}



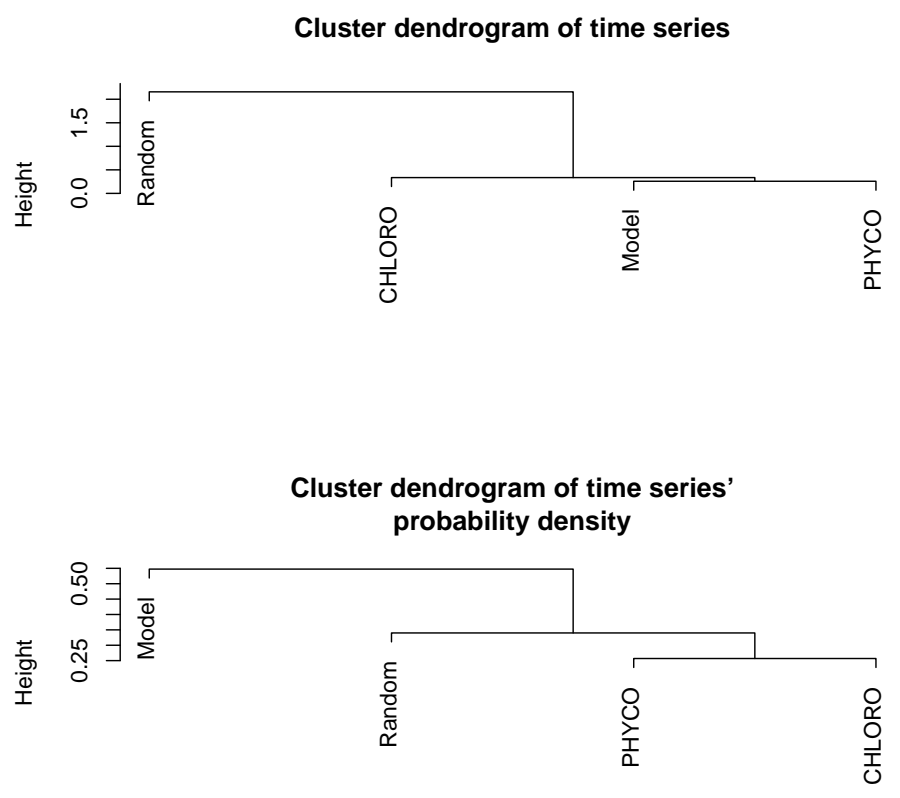

Figure 12: Hierarchical clustering of time series and corresponding probability density, based on distance matrix measured by TSB. 


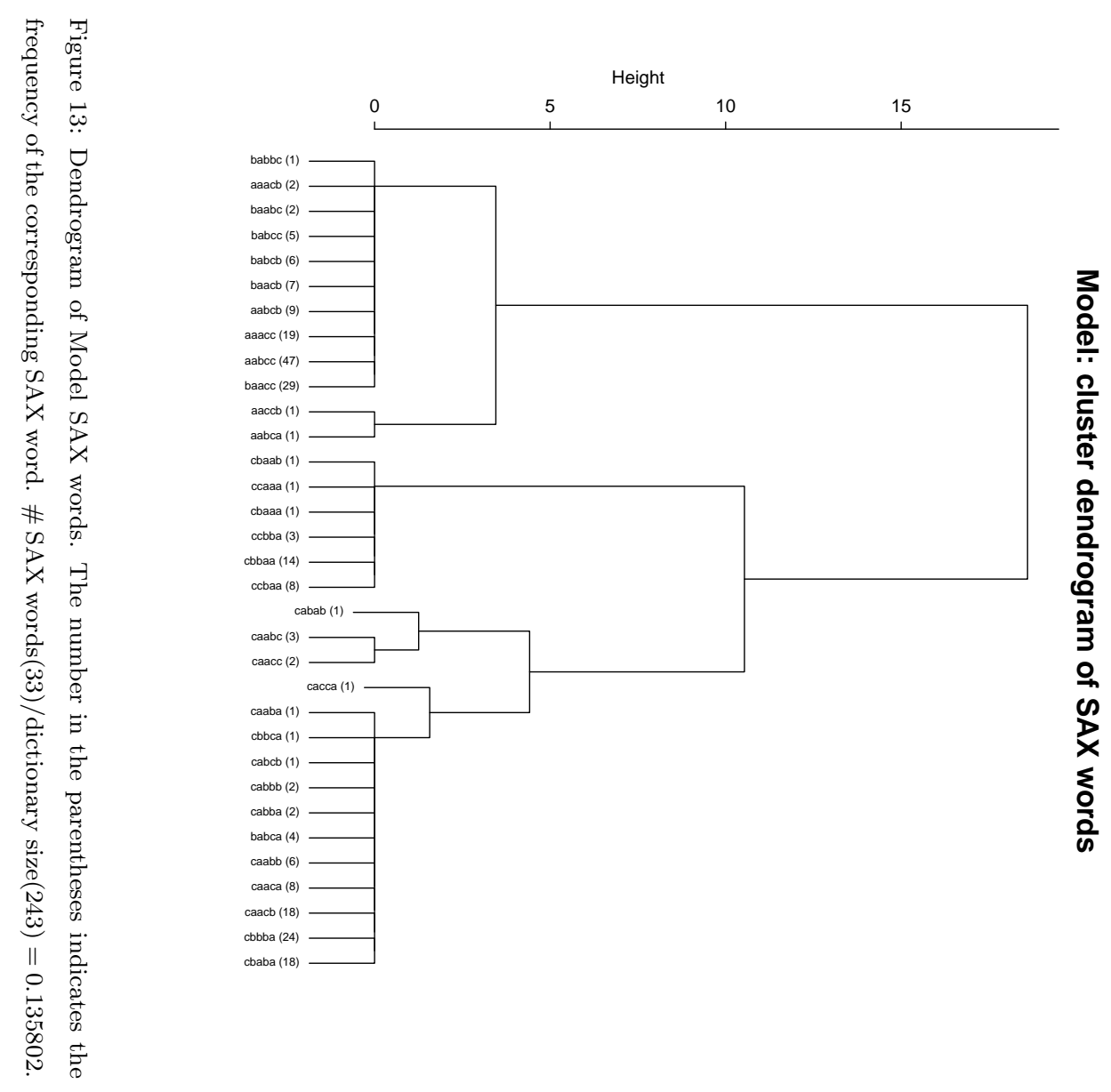




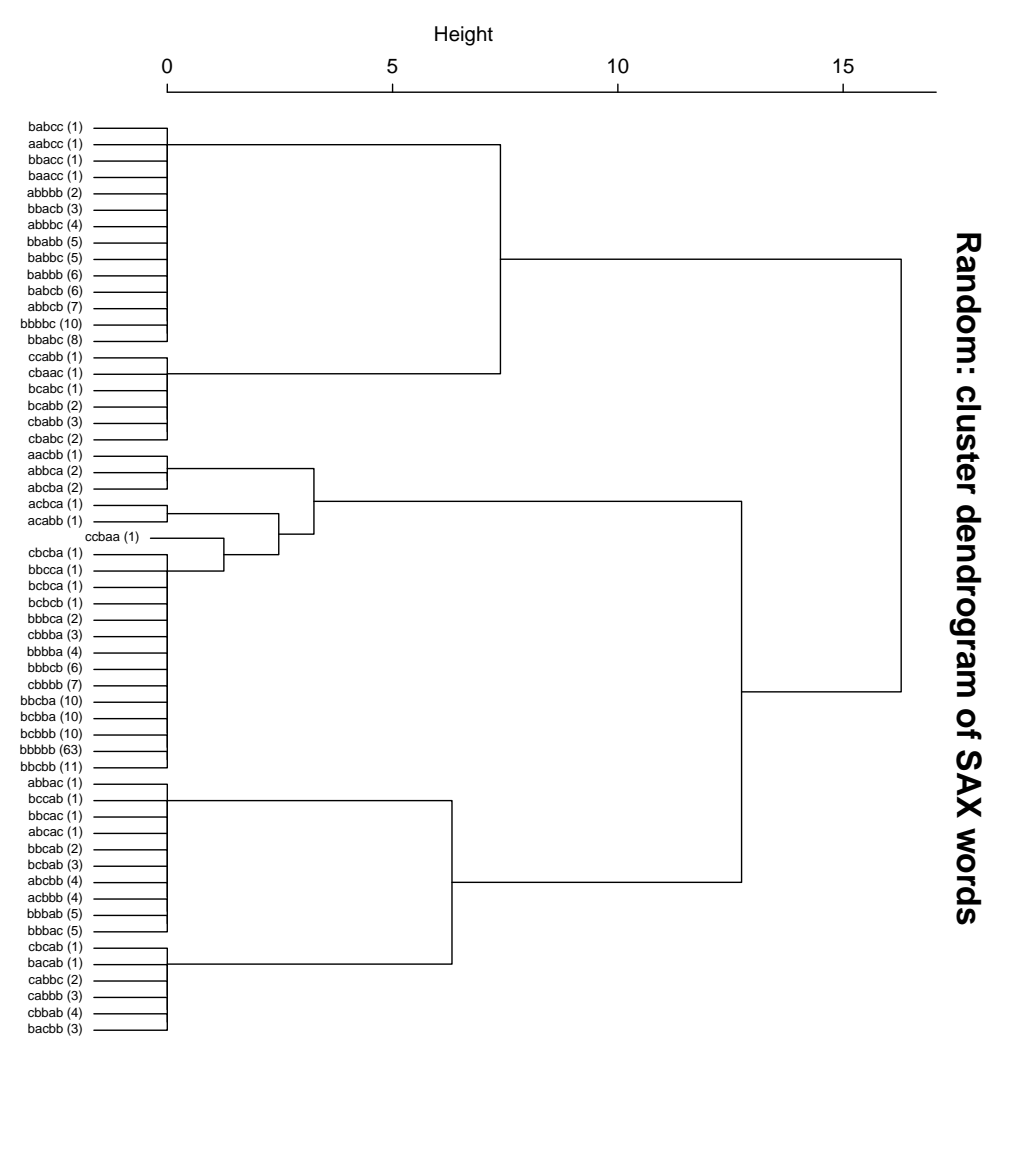



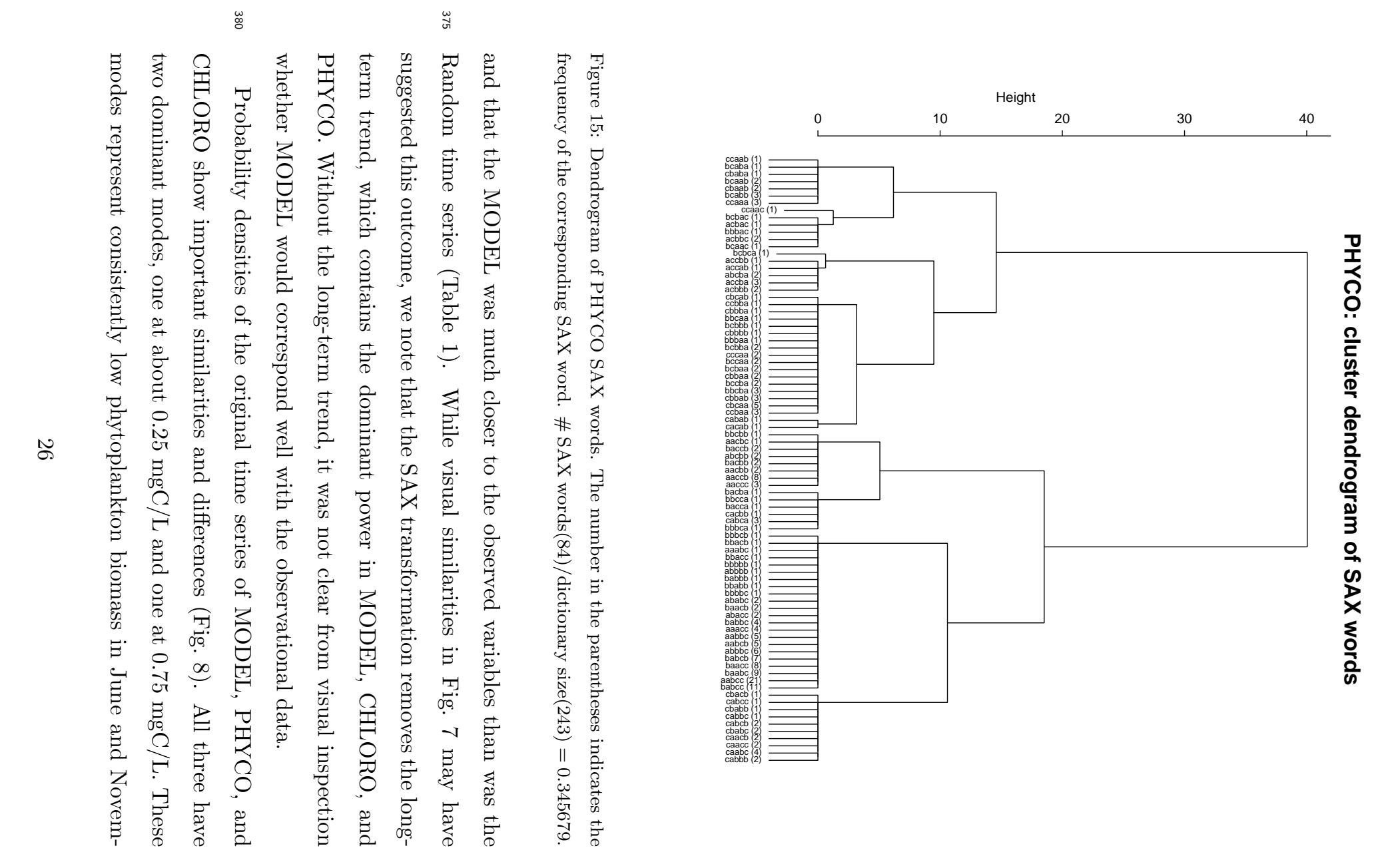

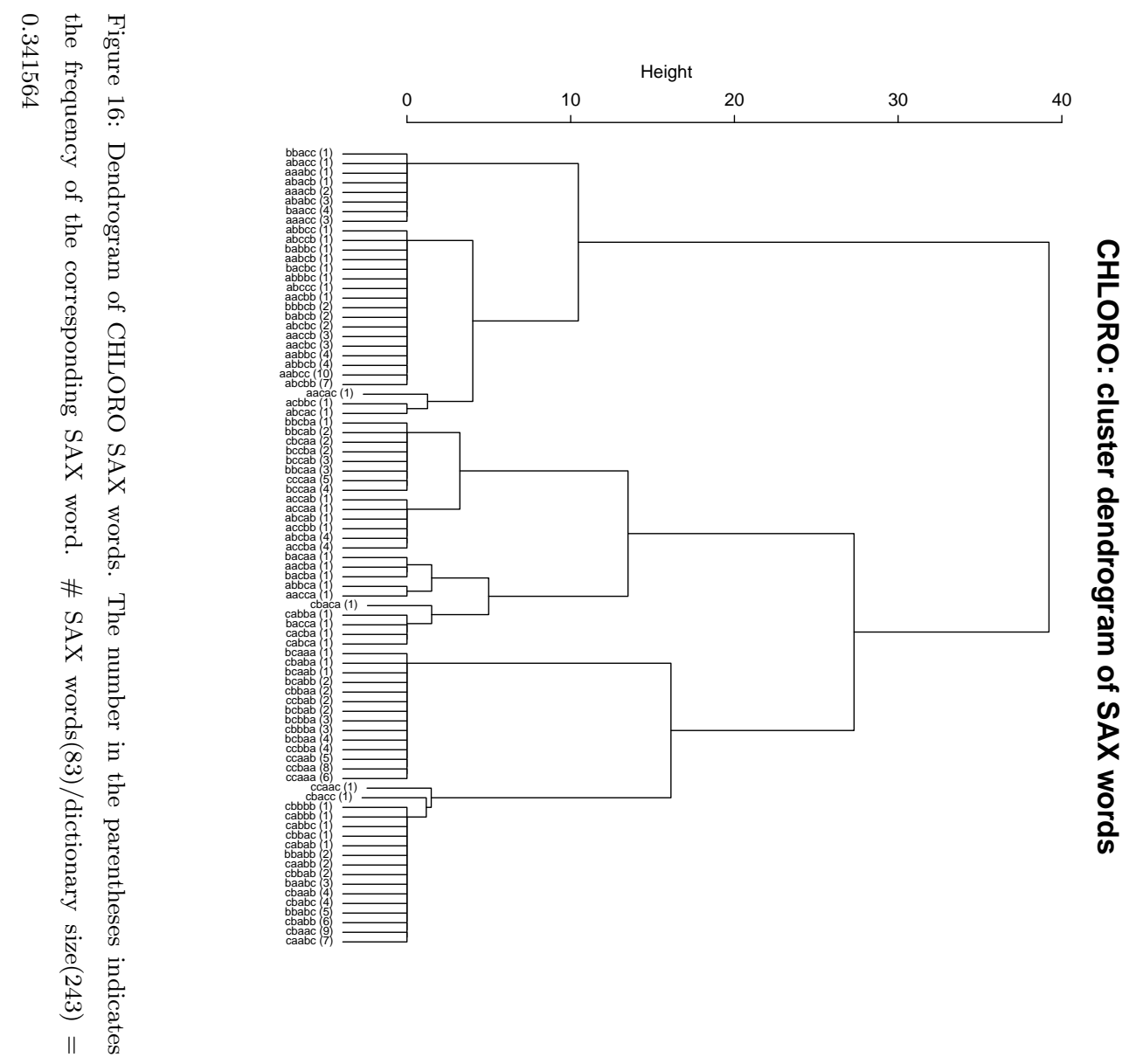


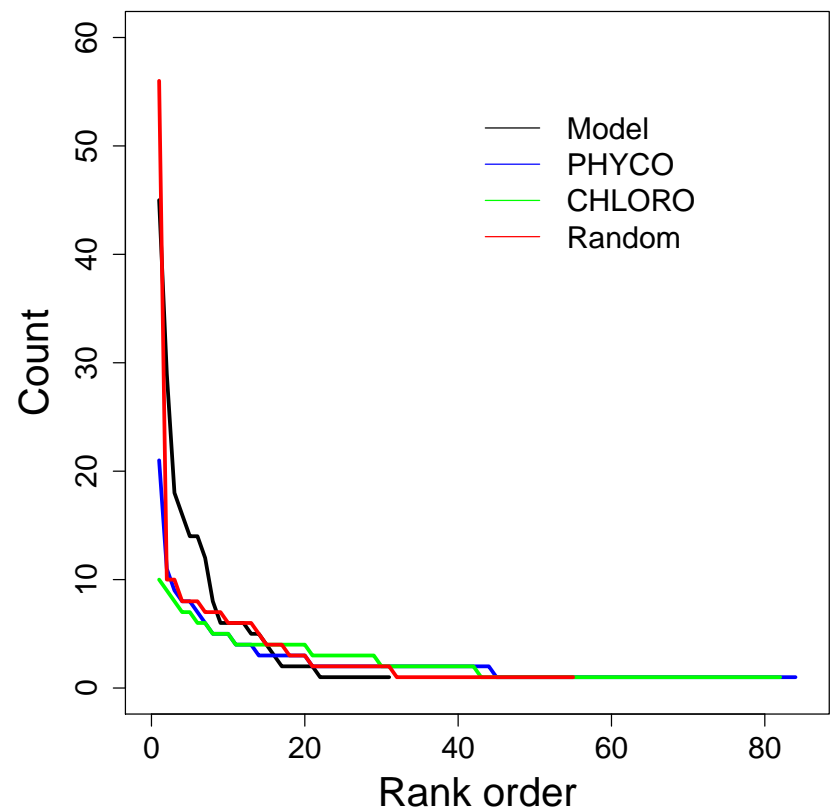

Figure 17: Count distribution of SAX words of Model, PHYCO, CHLORO and Random

ber and consistently high biomass from July through September (Fig. 7). Note, however, the long tails on the PHYCO and CHLORO distributions (Fig. 8), suggesting the rare occurrence of high phytoplankton biomass. We might interpret these rare events as phytoplankton blooms. While MODEL and PHYCO correspond well for the two dominant modes, the importance of those modes is decreased in the SAX transformation, and the weight of long tails increased. The consequence is that CHLORO and PHYCO distributions are more similar than either variables similarity with MODEL (Table 2).

\section{Related Work}

Much attention has been given to the representation of time series for ease and efficiency of mining, including the Discrete Fourier Transform (DFT) [12], the Discrete Wavelet Transform (DWT) 11], Piecewise Linear, and Piecewise Constant models (PAA) 14], (APCA) [29, 14], and Singular Value Decomposi- 
tion (SVD) 14]. There are additionally a great number of papers on discretizing (e.g., symbolizing, tokenizing and quantizing) time series [30, 13]; interested readers should refer to [31] for an extensive survey. However, none of the techniques allows a distance measure that lower bounds a distance measure defined on the original time series. Superior to the above approach, Symbolic Aggregate approXimation or SAX [4], a symbolic representation of time series, allows dimensionality and volume reduction, and a distance measure to be defined on the symbolic approach that lower bound corresponding distance measures defined on the original series.

\section{Conclusion}

In this paper, we leverage state-of-the-art time series mining techniques based on symbolic representation as well as the power of high performance computing (HPC) to facilitate knowledge discovery in lake ecology. Developed as a set of $\mathrm{R}$ libraries, we enable motif discovery and anomaly detection within a single lake time series, and relationship study amongst multiple lake time series through distance metrics, clustering and classification. To efficiently handle the large-scale sensor data and computation intensive simulation, we provision web services to launch $\mathrm{R}$ tools remotely to HPC resources.

Experimental results using both observational and simulated lake data demonstrate the effectiveness of the text mining routines in exposing information hidden in time series data, even when the volume of the SAX transformation was only $1 \%$ of the original data. Because of repeated pattern in the data, the model, CHLORO, and PHYCO time series had 33, 59, and 84 distinct words, respectively, out of a total vocabulary size of 243 words. Similarity between words within each vocabulary indicate that 7-11 groups of similar words account for the variability in the data. While patterns in MODEL matched those in PHYCO better than they matched patterns in CHLORO, MODEL failed to reproduce the long tail of the distribution, indicating that MODEL failed 
vocabulary, when compared with those from PHYCO and CHLORO, indicates that the MODEL does not recreate specific patterns through time. Further work is needed to investigate the importance of those difference and their implications for lake dynamics. For example, differences in motifs between MODEL, PHYCO, and CHLORO may represent mechanisms controlling fluorescence that are not in the model. These include pigment quenching during daytime, light history of phytoplankton, and temperature effects on fluorescence.

Although the mining algorithms are parameter light, it is still desirable to automate the parameter selection process (i.e., choosing the optimal parameters including alphabet size, word size, sliding window size, anomaly threshold, and etc.). This eases the use of the mining tools, allowing lake ecologists to focus more fully on their own research. One possible solution is to optimize the parameters by formulating an objective function (e.g., the classification accuracy). There are ample optimization algorithms available from which to choose; this is future work.

The SAX transformation is a unique approach to studying lake time series data by identifying patterns as data features and then using those features to make comparisons between variables, both observed and modeled. We envision SAX being incorporated into formal model evaluation, using the SAX distance 445 metric as an objective function to be minimized during model calibration; thus, enabling fit based on the patterns a model produces. We expect that the utility of in-lake comparisons would translate to between-lake comparisons, as well. As the corpus of lake sensor data grows, quickly mining all variables for patterns has intriguing possibilities. For example, lakes might be classified according to their pattern occurrences through time, their distribution of patterns, or the patterns inherent in their distributions. This capacity may enable us to address questions, such as: Might tomorrow's pattern in phytoplankton change from recent patterns (i.e., what is the likely pattern sequence through time)? How well can we classify and predict lake behavior based on its collection of 455 patterns? Do lakes of differing trophic states have differing pattern signatures? What is the probability of an extreme phytoplankton growth event (i.e., what 
proportion of the patterns have an upward step change)? The use of machine learning techniques, such as SAX, provide rich new approaches to explore the information content of ecosystem data.

\section{Acknowledgment}

This research is the result of a collaboration between the Global Lake Ecological Observatory Network (GLEON) 32] and Pacific Rim Application and Grid Middleware Assembly (PRAGMA) [33] communities. This research is funded in part through a grant from PRAGMA, grant \# OCI-1234983 and a grant by ${ }_{65}$ National Science Foundation Cyber-Enabled Discovery and Innovation (NSF CDI), under \# DEB-0941510. Any opinions, findings, conclusions or recommendations expressed in this material are those of the author(s) and do not necessarily reflect the views of the National Science Foundation.

\section{References}

470 [1] J. H. Porter, P. C. Hanson, C.-C. Lin, Staying afloat in the sensor data deluge, Trends in Ecology and Evolution 27 (2) (2012) 121-129. doi:10.1016/j.tree.2011.11.009

[2] B. A. Pellerin, B. A. Stauffer, D. A. Young, D. J. Sullivan, S. B. Bricker, M. R. Walbridge, G. A. C. Jr., D. M. Shaw, Emerging tools for continuous nutrient monitoring networks: Sensors advancing science and water resources protection, Journal of the American Water Resources Association (JAWRA) 52 (4) (2016) 993-1008. doi:10.1111/1752-1688.12386.

[3] C. Gries, M. Gahler, P. C. Hanson, T. Kratz, E. Stanley, Information management at the north temperate lakes long-term ecological research site - successful support of research in a large, diverse, and long running projec, Ecological Informatics 36 (2016) 201-208. doi:http://dx.doi.org/10.1016/j.ecoinf.2016.08.007. 
[4] J. Lin, E. Keogh, S. Lonardi, B. Chiu, A symbolic representation of time series, with implications for streaming algorithms, in: Proceedings of the 8th ACM SIGMOD workshop on Research Issues in Data Mining and Knowledge Discovery, Washington D.C., USA, 2003, pp. 2-11.

[5] E. P. Kasten, P. K. McKinley, S. H. Gage, Automated ensemble extraction and analysis of acoustic data strems, in: Proceedings of the 27th International Conference on Distributed Computing Systems Workshops (ICDCSW'07), Washington D.C., USA, 2007.

[6] Y. Liu, D. Bao, Z. Yang, Application research of a new symbolic approximation method - sax in time series mining, Computer Engineering and Applications 42 (27).

[7] J. Lin, E. Keogh, L. Wei, S. Lonardi, Experiencing sax: a novel symbolic representation of time series, Data Mining and Knowledge Discovery 15 (2) (2007) 107-144. doi:10.1007/s10618-007-0064-z.

[8] J. Lin, E. Keogh, S. Lonardi, P. Patel, Finding motifs in time series, in: Proceedings of the 2nd Workshop on Temporal Data Mining, co-located with SIGKDD’02, Edmonton, Alberta, Canada, 2002, pp. 53-68.

[9] E. Keogh, S. Lonardi, C. A. Ratanamahatana, Towards parameter-free data mining, in: Proceedings of the 10th ACM SIGKDD International Conference on Knowledge Discovery and Data Mining, Seattle, WA, USA, 2004, pp. 206-215.

[10] N. Kumar, V. Nishanth, L. Eamonn, K. Stefano, L. Chotirat, A. Ratanamahatana, L. Wei, Time-series bitmaps: A practical visualization tool for working with large time series databases, in: Proceedings of 2005 SIAM International Conference on Data Mining, 2005, pp. 531-535.

[11] K.-P. Chan, A.-C. Fu, Efficient time series matching by wavelets, in: Proceedings of the 15th International Conference on Data Engineering, Sydney, Austrialia, 1999, pp. 126-133. 
[12] C. Faloutsos, M. Ranganathan, Y. Manolopoulos, Fast subsequence matching in time-series databases, in: Proceedings of the 1994 ACM SIGMOD International Conference on Management of Data, Minneapolis, MN, USA, 1994, pp. 419-429.

[13] Y.-W. Huang, P. S. Yu, Efficient time series matching by wavelets, in: Proceedings of the 5th ACM SIGKDD International Conference on Knowledge Discovery and Data Mining, San Diego, CA, USA, 1999, pp. 282-286.

[14] E. Keogh, K. Chakrabarti, M. Pazzani, S. Mehrotra, Locally adaptive dimensionality reduction for indexing large time series databases, in: Proceedings of the 2001 ACM SIGMOD International Conference on Management of Data, Santa Barbara, CA, USA, 2001, pp. 151-162.

[15] E. Keogh, M. J. Pazzani, An enhanced representation of time series which allows fast and accurate classification, clustering and relevance feedback, in: Proceedings of the 4th ACM SIGKDD International Conference on Knowledge Discovery and Data Mining, 1998, pp. 239-243.

[16] B.-K. Yi, C. Faloutsos, Fast time sequence indexing for arbitrary lp norms, in: Proceedings of the 26th International Conference on Very Large Databases, Cairo, Egypt, 2000, pp. 385-394.

[17] M. L. Marx, R. J. Larsen, An Introduction to Mathematical Statistics and Its Applications, 2nd Edition, Pearson/Prentice Hall, 1986.

[18] J. Shieh, E. Keogh, isax: Indexing and mining terabyte sized time series, in: Proceedings of the 14th ACM SIGKDD International Conference on Knowledge Discovery and Data Mining, Las Vegas, Nevada, USA, 2008, pp. $623-631$.

[19] Big red ii at indiana university, https://kb.iu.edu/d/bcqt.

[20] B. D. Bojanov, H. Hakopian, B. Sahakian, Spline functions and multivariate interpolations, Springer Science and Business Media 248. 
[21] D. J. Berndt, J. Clifford, Using dynamic time warping to find patterns in time series, in: Proceedings of the 1994 KDD Workshop, Seattle, Washington, USA, 1994, pp. 359-370.

[22] E. Keogh, S. Kasetty, On the need for time series data mining benchmarks: a survey and empirical demonstration, in: Proceedings of the 8th ACM SIGKDD International Conference on Knowledge Discovery and Data Mining, Edmonton, Alberta, Canada, 2002, pp. 102-111.

545 [23] M. Li, P. Vitányi, An introduction to kolmogorov complexity and its applications, Springer Science and Business Media.

[24] D. W. Schindler, Evolution of phosphorus limitation in lake, Science 195 (4275) (1977) 260-262.

[25] U. Sommer, Z. M. Gliwicz, W. Lampert, A. Duncan, The peg model of a seasonal succession of planktonic events in fresh waters, Arch. Hydrobiol 106 (4) (1986) 433-471.

[26] M. R. Hipsey, D. P. Hamilton, P. C. Hanson, et al., Predicting the resilience and recovery of aquatic systems: A framework for model evolution within environmental observatories, Water Resources Research 51 (9) (2015) 70237043.

[27] E. L. Kara, P. C. Hanson, D. P. Hamilton, M. Hipsey, K. D. McMahon, J. Read, L. Winslow, J. Dedrick, K. Rose, C. C. Carey, S. Bertilsson, D. M. Marques, L. Beversdorf, T. Miller, C. Wu, Y. F. Hsieh, E. Gaiser, T. K. Kratz, Time-scale dependence in numerical simulations: Assessment of physical, chemical, and biological predictions in a stratified lake at temporal scales of hours to months, Environmental Modelling \& Software 35 (2012) 104-121. doi:10.1016/j.envsoft.2012.02.014.

[28] M. R. Hipsey, L. C. Bruce, D. P. Hamilton, Glm: general lake model. model overview and user information, in: The University of Western Australia Technical Manual, Perth, Australia, 2013. 
[29] P. Geurts, Pattern extraction for time series classification, Principles of Data Mining and Knowledge Discovery (2001) 115-127.

[30] H. André-Jönsson, D. Z. Badal, Using signature files for querying timeseries data, Principles of Data Mining and Knowledge Discovery (1997) $211-220$.

[31] S. C. Daw, C. E. A. Finney, E. R. Tracy, A review of symbolic analysis of experimental data, Review of Scientific Instruments 74 (2) (2003) 915-930.

[32] Global lake ecological observatory network, http://gleon.org/.

[33] Pacific rim application and grid middleware assembly, http://www.pragma-grid.net/. 